\title{
Drug detection in biological specimens: recent colorimetric methods
}

\author{
Samin Hamidi*,1 \\ ${ }^{1}$ Food \& Drug Safety Research Center, Tabriz University of Medical Sciences, Tabriz 51664, Iran \\ * Author for correspondence: Tel: +98 41 34421924; Fax: +98 41 34422438; hamidisamin@gmail.com
}

First draft submitted: 11 October 2017; Accepted for publication: 6 December 2017; Published online: 15 January 2018

Keywords: bioanalysis $\bullet$ colorimetry $\bullet$ metal nanoparticles

Recently, the detection approaches based on the metal nanoparticles (NPs) have attracted more and more attention due to their great extinction coefficient and strong surface plasma resonance properties. This report focuses on the present status of drug detection with metal NPs as ultrasmall labels in colorimetric-based methods. Although progresses have been emerged in drug detection, much work remains to be done to overcome the great challenges on sample preparation of complicated biological fluids. Plasmonic NPs can be combined with other existing functional groups to produce frameworks with novel multifunctional surface plasmon resonance for generating optical platform in drug detection. With their continued progress, we believe that making high quality and multifunctional plasmonic materials will play an important role in the drug detection and quantification in biological samples.

Many important disciplines involving human health are dealing with the analysis of drugs present in living organisms or biological fluids. Biological samples are difficult to handle owing to their inherent complexity and, sometimes, sample preparation processes such as tryptic digestion make them even more complex. However, in the case of simpler matrices such as exhaled breath condensate, the direct injection of sample to analytical instruments is possible [1-3]. This complexity hinders the reliable analysis in two ways. First, most biological fluids are not amendable with analytical instruments since all biological samples consist of a vast range of ions, proteins, lipids, carbohydrates and so on, and cannot be injected to analytical devices. Second, highly abundant interfering compounds disturb the identification and quantification of trace analytes at subnanogram per milliliter levels.

A bioanalytical strategy consists of two integral stages:

- Sample preparation (treatment/cleanup/extraction) of the target analyte from its complex biological matrix;

- Separation, detection and quantification of the analyte.

Common technologies for drug determination in body fluids including ELISA, chromatographic and electrophoretic-based methods suffer from several limitations in bioanalytical laboratories. Owing to the complex matrix of most biological specimens, they rely on time- and labor-consuming sample purification. Moreover, sophisticated instruments such as MS are not affordable by many of the laboratories and require effective extraction approaches [4-8]. It is noteworthy that the sensitivity of some of these technologies is not adequate for detecting the required levels of target analyte. Therefore, there is still a great demand to develop inexpensive, fast, user-friendly and easy-to-operate technologies.

The association of nanotechnology with medical treatment, including therapeutic drug monitoring, pharmacokinetic and pharmacodynamic studies has provided a new concept in drug detection. Label-free detection systems with little to no sample preparation is more appreciated [9]. Colorimetric methods use the intrinsic advantages of colorful species upon the addition of a certain analyte. In this regard, the use of noble metal NPs (in particular gold and silver) for colorimetric purposes have received great attention.

\section{Metallic NPs}

Recently, the detection approaches based on the metallic NPs have attracted attention due to their great extinction coefficient and strong surface plasma resonance properties in the visible region [10]. These materials are able to provide the sensitive and selective detection systems. Metallic NPs often act as effective detection material as they 
can be easily probed by optical approach due to their local surface plasmon resonance (LSPR) property. They often show high absorption coefficients, and therefore they possess a high level of sensitivity in optical based approaches. One of the important aspects in NPs is the surface charge generated by the capping agent (stabilizer), because it can determine the stability and behavior of the NP upon changing microenvironment surrounding and molecular interactions [11,12].

\section{Gold NPs}

Gold NPs (AuNPs) show interesting physical and chemical properties that make them excellent materials for the fabrication of novel chemical and biological sensors. First, AuNPs can be prepared in a straightforward route and remain stable. Second, AuNPs can be readily modified by varying their size, morphology and the surrounding chemical environment. For instance, the interaction between the recognition element and the analyte can tune physicochemical properties of AuNPs, such as plasmon resonance absorption, optical properties, conductivity biocompatibility and redox behavior which in turn can provide a suitable platform for calculating the detectable signal [10,13-14]. Each of these extraordinary characteristics of AuNPs has encouraged researchers to develop novel sensing strategies with specific sensitivity, stability and selectivity for target analyte. As colorimetric probes, AuNPs have attracted great interests which can directly detect analytes by following the color change, using spectrophotometric method or even with naked eyes. Apparently, this method is of prime importance as no complicated instruments are needed in the detection procedures [15,16]. Moreover, the color change shows great sensitivity to the size, shape, capping agent, medium refractive index and state of AuNPs.

Electron-rich nitrogen-containing agent has been used to stabilize AuNPs. The lone pair of the nitrogen group (such as primary amines) is able to chemically adsorb onto the surface of the AuNPs, with the alkyl group preventing agglomeration via steric stabilization [14,17]. Clonazepam is a drug of the benzodiazepines and is found in very small amounts. To improve the detection limit, nanotechnology has become a powerful tool to trace small amounts of drugs from the complex samples. Clonazepam could interact with melamine through hydrogen bonding interactions as it possesses $\mathrm{NO}_{2},-\mathrm{NH}-$ and $-\mathrm{Cl}$ functional groups. So, clonazepam would act as a molecular bridge inducing the aggregation of AuNPs. The colorimetric detection of drug concentrations was in the range of $10 \times 10^{-8}$ $10 \times 10^{-9} \mathrm{M}$. This method is an excellent platform for on-site and real time screening of clonazepam from different body specimens [18]. 4-amino-3-hydrazino-5-mercapto-1,2,4-triazol (AHMT) fabricated AuNPs (AHMT-AuNPs) was used as an optical probe for detection of dopamine in human serum and urine samples in the range of $0.20-1.10 \mu \mathrm{M}$. Dopamine has three sites to form hydrogen bonds therefore, dopamine was linked between the AHMT-AuNPs via hydrogen bonding and caused to aggregation of particles and color change [19].

Clenbuterol is a drug used to treat bronchial asthma. It is also a kind of adrenal neural stimulant that is involved in protein synthesis, which can prompt the transformation and the decomposition of fat [20]. Kang et al., prepared AuNPs by using sodium citrate reduction method and citrate-stabilized AuNPs were decorated with cysteamine. In this method, citric acid acts as both reducing and stabilizing material and generates AuNPs with diameters around $20 \mathrm{~nm}$. The $-\mathrm{NH}_{2}$ group of cysteamine can connect with $-\mathrm{OH},-\mathrm{Cl}$ and $-\mathrm{NH}_{2}$ of clenbuterol through hydrogen bonding and then AuNPs aggregated quickly, causing to the color change of the solution from wine red to blue grey, due to the change of SPR absorption peak and intensity. They implied that this sensor has been successfully applied to determine clenbuterol in blood, which may lead to a new way for naked-eye clenbuterol probe in real samples [21].

\section{Silver NPs}

Silver NPs (AgNPs) have attracted great interest due to their strong and tunable optical characteristics originating from LSPR. Spherical AgNPs in $10 \mathrm{~nm}$ size range have a unique LSPR absorption band with a maximum of around $390 \mathrm{~nm}$ [22]. Also the position of peak depends on NPs' size, shape and local dielectric environment [23].

NPs could be associated with the organic ligand as a stabilizer agent. Folic acid contains carboxylic acid and amino group, those can coordinate $\mathrm{Ag}^{+}$ions with good geometry [24]. 6-Mercaptopurine is a sulfur analog of adenine that was used as an anticancer and an immunosuppressive agent [25]. Folic acid modified AgNPs were used as probes in colorimetric determination of 6-mercaptopurine in the range of 20-1000 nM in urine samples [26]. The mechanism of action is relied on oxidation of $\mathrm{Ag}^{0}-\mathrm{Ag}^{+}$ion, resulting in a blue-shift in the SPR peak and a color change from yellow to colorless. Various agents have been employed as reducing agents and stabilizers for preparation of AgNPs. Among them, carbon-based nanomaterials have attracted great attention in this discipline. Carbon quantum dots (CQDs) are carbon NPs with interesting physicochemical properties. A facile synthesis of CQD/Ag nanocomposites 
using glucose-based CQDs as both reducing and stabilizing materials. Methimazole is one of the major drugs for treatment of hyperthyroidism. These nanocomposites exhibit a unique color change from yellow to red in the presence of trace amounts of methimazole in urine samples [27]. Chavada's group proposed a colorimetric assay for the determination of the deferiprone from plasma samples using AgNPs fabricated with pyrophosphate groups (Pyro-AgNPs). Deferiprone was determined in the range of 6.0-100 $\mu \mathrm{M}$ using spectrophotometer [28].

Recently, investigations have been focused toward the design of biosensors based on DNA aptamer for drug detection. A nonaggregated Au@Ag-based colorimetric strategy was implemented for visual sensing platform to detect methamphetamine and cocaine in urine samples in the range of $0.5-200 \mathrm{nM}$. The biosensor consisted of a specific single stranded DNA sequences functionalized with $\mathrm{Au} @ \mathrm{Ag}$, a capture probe conjugated with superparamagnetic magnetic beads and an illicit drug-binding DNA aptamer [29].

Lidocaine is an aromatic amide which is widely used as local anesthetics. Menon's group reported the use of a smart phone as a portable and cost-effective colorimetric sensor for lidocaine hydrochloride determination in the range of $0.1-4.5 \mathrm{ng} / \mathrm{ml}$ in blood and vitreous humor samples. Using the citrated capped AgNPs probe as the aggregation of AgNPs occur rapidly in the presence of lidocaine due to the electrostatic and hydrogen-bonding interaction between them which results in color change from yellow to red [30].

\section{Conclusion \& future perspective}

This report focuses on the present status of drug detection with metal NPs as ultrasmall labels in colorimetricbased methods. Although significant progresses have emerged in drug detection, much work remains to be done to overcome the great challenges on sample preparation of complicated biological fluids. Plasmonic NPs can be combined with other functional materials to generate nanostructures with novel multifunctional SPR for achieving optical drug detection. Plasmonic NPs can be combined with other existing functional groups to produce frameworks with novel multifunctional SPR for generating optical platform in drug detection. With their continued progress, we believe that making high quality and multifunctional plasmonic materials will play an important role in the drug detection and quantification in biological samples. Moreover, the incorporation of NPs in devices provides portable, easy to use and inexpensive sensors, due to the ease of miniaturization of both the reagents and the transduction setup. Medical professionals are increasingly exploring personalized healthcare as a tool of improving healthcare systems of the future. Point-of-care (POC) devices and 'lab-on-a-chip' setup aim to make this a reality, with such developments in the drug detection domain. Yet, clinical use of such colorimetric diagnostics has not revealed with the number of commercially available testing platforms. Despite this, there is continued progress on laboratories to miniaturize these tests, being the POC diagnostics to enter clinical care and research. They will likely serve as commercially available vehicles for addressing POC tests in the near future.

\section{Financial \& competing interests disclosure}

The author has no relevant affiliations or financial involvement with any organization or entity with a financial interest in or financial conflict with the subject matter or materials discussed in the manuscript. This includes employment, consultancies, honoraria, stock ownership or options, expert testimony, grants or patents received or pending, or royalties.

No writing assistance was utilized in the production of this manuscript.

\section{References}

1. Hamidi S, Khoubnasabjafari M, Ansarin K, Jouyban-Gharamaleki V, Jouyban A. Direct analysis of methadone in exhaled breath condensate by capillary zone electrophoresis. Curr. Pharm. Anal. 12(2), 137-145 (2016).

2. Hamidi S, Khoubnasabjafari M, Ansarin K, Jouyban-Gharamaleki V, Jouyban A. Chiral separation of methadone in exhaled breath condensate using capillary electrophoresis. Anal. Methods 9(15), 2342-2350 (2017).

3. Jouyban A, Khoubnasabjafari M, Ansarin K et al. Methadone concentrations in exhaled breath condensate, serum and urine of patients under maintenance treatment. Iran. J. Pharm. Res. 16(4), 1621-1630 (2017).

4. Hamidi S, Soltani S, Jouyban A. A dispersive liquid-liquid microextraction and chiral separation of carvedilol in human plasma using capillary electrophoresis. Bioanalysis 7(9), 1107-1117 (2015).

5. Hamidi S, Jouyban A. Capillary electrophoresis with UV detection, on-line stacking and off-line dispersive liquid-liquid microextraction for determination of verapamil enantiomers in plasma. Anal. Methods 7(14), 5820-5829 (2015).

6. Hamidi S, Jouyban A. Preconcentration approaches combined with capillary electrophoresis in bioanalysis of chiral cardiovascular drugs. Pharm. Sci. 21 229-243 (2015). 
7. Jouyban A, Hamidi S. Dispersive micro solid phase extraction using carbon-based adsorbents for sensitive determination of verapamil in plasma samples coupled with CE. J. Sep. Sci. 40(16), 3318-3326 (2017).

8. Hamidi S, Alipour-Ghorbani N. Liquid phase microextraction of biomarkers: a review on current methods. J. Liq. Chromatogr. Relat. Technol. 40(16), 853-861 (2017).

9. Homola J. Surface plasmon resonance sensors for detection of chemical and biological species. Chem. Rev. 108(2), $462-493$ (2008).

10. Saha K, Agasti SS, Kim C, Li X, Rotello VM. Gold nanoparticles in chemical and biological sensing. Chem. Rev. 112(5), 2739-2779 (2012).

11. Lynch I, Dawson KA. Protein-nanoparticle interactions. Nano Today 3(1), 40-47 (2008).

12. Gebauer JS, Malissek M, Simon S et al. Impact of the nanoparticle-protein corona on colloidal stability and protein structure. Langmuir 28(25), 9673-9679 (2012).

13. Xiao L, Wildgoose GG, Compton RG. Sensitive electrochemical detection of arsenic (III) using gold nanoparticle modified carbon nanotubes via anodic stripping voltammetry. Anal. Chim. Acta 620(1), 44-49 (2008).

14. Chi H, Liu B, Guan G, Zhang Z, Han MY. A simple, reliable and sensitive colorimetric visualization of melamine in milk by unmodified gold nanoparticles. Analyst 135(5), 1070-1075 (2010).

15. Jiang Y, Zhao H, Zhu N, Lin Y, Yu P, Mao L. A simple assay for direct colorimetric visualization of trinitrotoluene at picomolar levels using gold nanoparticles. Angew. Chem. Int. Ed. Engl. 120(45), 8729-8732 (2008).

16. Jiang Y, Zhao H, Lin Y, Zhu N, Ma Y, Mao L. Colorimetric detection of glucose in rat brain using gold nanoparticles. Angew. Chem. Int. Ed. Engl. 122(28), 4910-4914 (2010).

17. Zhang X, Zhao H, Xue $\mathrm{Y}$ et al. Colorimetric sensing of clenbuterol using gold nanoparticles in the presence of melamine. Biosens. Bioelectron. 34(1), 112-117 (2012).

18. Lodha A, Pandya A, Sutariya PG, Menon SK. Melamine modified gold nanoprobe for "on-spot" colorimetric recognition of clonazepam from biological specimens. Analyst 138(18), 5411-5416 (2013).

19. Feng JJ, Guo H, Li YF, Wang YH, Chen WY, Wang AJ. Single molecular functionalized gold nanoparticles for hydrogen-bonding recognition and colorimetric detection of dopamine with high sensitivity and selectivity. ACS Appl. Mater. Inter. 5(4), 1226-1231 (2013).

20. Fu X, Chu Y, Zhao K, Li J, Deng A. Ultrasensitive detection of the $\beta$-adrenergic agonist brombuterol by a SERS-based lateral flow immunochromatographic assay using flower-like gold-silver core-shell nanoparticles. Microchim. Acta 184(6), 1711-1719 (2017).

21. Kang J, Zhang Y, Li X, Miao L, Wu A. A rapid colorimetric sensor of clenbuterol based on cysteamine-modified gold nanoparticles. ACS Appl. Mater. Inter. 8(1), 1-5 (2015).

22. Jain PK, Huang X, El-Sayed IH, El-Sayed MA. Noble metals on the nanoscale: optical and photothermal properties and some applications in imaging, sensing, biology and medicine. Acc. Chem. Res. 41(12), 1578-1586 (2008).

23. Kelly KL, Coronado E, Zhao LL, Schatz GC. The optical properties of metal nanoparticles: the influence of size, shape and dielectric environment. J. Phys. Chem. B 107(3), 668-677 (2003).

24. Su D, Yang X, Xia Q et al. Folic acid functionalized silver nanoparticles with sensitivity and selectivity colorimetric and fluorescent detection for $\mathrm{Hg}_{2}{ }^{+}$and efficient catalysis. Nanotechnology 25(35), 355702 (2014).

25. Sahasranaman S, Howard D, Roy S. Clinical pharmacology and pharmacogenetics of thiopurines. Eur. J. Clin. Pharmacol. 64(8), 753-767 (2008)

26. Rawat KA, Singhal RK, Kailasa SK. One-pot synthesis of silver nanoparticles using folic acid as a reagent for colorimetric and fluorimetric detections of 6-mercaptopurine at nanomolar concentration. Sensor. Actuator. B Chem. 249, 30-38 (2017).

27. Amjadi M, Hallaj T, Asadollahi H, Song Z, De Frutos M, Hildebrandt N. Facile synthesis of carbon quantum dot/silver nanocomposite and its application for colorimetric detection of methimazole. Sensor. Actuator. B Chem. 244, 425-432 (2017).

28. Chavada VD, Bhatt NM, Sanyal M, Shrivastav PS. Pyrophosphate functionalized silver nanoparticles for colorimetric determination of deferiprone via competitive binding to Fe (III). Microchim. Acta 184(10), 42034208 (2017).

29. Mao K, Yang Z, Li J, Zhou X, Li X, Hu J. A novel colorimetric biosensor based on nonaggregated Au@ Ag core-shell nanoparticles for methamphetamine and cocaine detection. Talanta 175, 338-346 (2017).

30. Ansari N, Lodha A, Pandya A, Sutariya PG, Menon SK. Lab-on-phone citrate-capped silver nanosensor for lidocaine hydrochloride detection from a biological matrix. Anal. Methods 7(21), 9084-9091 (2015). 